\title{
Low-background prebunching system for heavy-ion beams at the Tokai radioactive ion accelerator complex
}

\author{
M. Okada, K. Niki, Y. Hirayama, N. Imai, H. Ishiyama, S. C. Jeong, I. Katayama, H. Miyatake, ${ }^{*}$ \\ M. Oyaizu, and Y.X. Watanabe \\ High Energy Accelerator Research Organization (KEK), Oho 1-1, Tsukuba, Ibaraki 305-0801, Japan \\ S. Arai \\ RIKEN Nishina Center, Wako 2-1, Saitama 351-0198, Japan \\ H. Makii and Y. Wakabayashi \\ Japan Atomic Energy Agency (JAEA), Shirakata Shirane 2-4, Tokai, Ibaraki 319-1195, Japan
}

(Received 22 June 2011; published 19 March 2012)

\begin{abstract}
A novel beam-bunching technique has been implemented at a heavy-ion linear accelerator facility by installing a compact two-gap prebuncher and a multilayer beam chopper. A pulsed beam of 2 to $4 \mathrm{MHz}$, having kinetic energy up to $1.1 \mathrm{MeV} / \mathrm{u}$, is realized by bunching a $2 \mathrm{keV} / \mathrm{u}$ continuous beam just upstream of the linac. Around $40 \%$ of the continuous beam particles are successively gathered in a single microbunch with a time width of around $15 \mathrm{~ns}$ in full width at one-tenth maximum. The number of background beam particles over $250 \mathrm{~ns}$ just before the bunched beam is well suppressed to less than $10^{-4}$ of the number of bunched particles. This technique has been adopted to generate intense $\alpha$-particle beams for nuclear astrophysics experiments.
\end{abstract}

DOI: 10.1103/PhysRevSTAB.15.030101

PACS numbers: 29.20.-c, 29.27.-a

\section{INTRODUCTION}

The Tokai radioactive ion accelerator complex (TRIAC) facility [1] is a radioactive ion beam (RIB) facility based on the postacceleration scheme, including two types of heavy-ion linear accelerators (linacs), i.e., a $26 \mathrm{MHz}$ split coaxial radio frequency quadrupole (SCRFQ)-type linac and a $52 \mathrm{MHz}$ interdigital $\mathrm{H}(\mathrm{IH})$-type linac, as shown in Fig. 1. This accelerator complex can accelerate heavy ions $(q / A \geq 1 / 10)$ from $2 \mathrm{keV} / \mathrm{u}$ to $1.1 \mathrm{MeV} / \mathrm{u}$ with high transport efficiency of around 85\% [2]. This region of acceleration energy is suitable for studying nuclear reactions under astrophysical circumstances such as elemental synthesis in supernova explosions. Thus far, some experiments have been performed using ${ }^{8} \mathrm{Li}$-RIBs $[3,4]$.

The $18 \mathrm{GHz}$ electron cyclotron resonance ion source (ECRIS) [5,6] located upstream of the linacs has made it possible for TRIAC to deliver heavy-ion beams of stable isotopes. A large emittance acceptance $(291 \pi \mathrm{mm} \mathrm{mrad})$ of the SCRFQ for a continuous beam makes it possible to accelerate such beam particles with intensities of several ten microamperes. Based on this characteristic of the TRIAC facility, researchers have proposed direct measurements of ${ }^{12} \mathrm{C}(\alpha, \gamma){ }^{16} \mathrm{O}$ stellar reactions using intense ${ }^{4} \mathrm{He}^{+}$beams.

\footnotetext{
*hiroari.miyatake@kek.jp

Published by the American Physical Society under the terms of the Creative Commons Attribution 3.0 License. Further distribution of this work must maintain attribution to the author(s) and the published article's title, journal citation, and DOI.
}

In this experiment, prompt $\gamma$ rays are measured directly by large volume $\mathrm{NaI}(\mathrm{Tl})$ detectors under the condition of low-background radiation [7], where $\alpha$ beams are bunched to define a time window of the fast coincidence between beam particles and detected events by the $\mathrm{NaI}(\mathrm{Tl})$ crystal. The emission of background $\gamma$ rays is mainly attributed to induced reactions of iodine in the detector and neutrons from $(\alpha, n)$ of the ${ }^{13} \mathrm{C}$ contaminant in the target. Hence, the fast coincidence technique coupled with the neutron time of flight has been adopted to suppress these $\gamma$ rays. Taking the neutron flight time and intensity of background $\gamma$ rays into account, the required bunch frequency of $\alpha$ beams is 2 to $4 \mathrm{MHz}$, the time width of the bunched beam is less than $20 \mathrm{~ns}$ in full width at tenth maximum (FWTM), and the number of background beam particles in the beam-off period, especially in the region preceding the bunched beam timing, is less than $10^{-4}$ of the number of bunched particles.

As the first stage accelerator of TRIAC, the SCRFQ linac can accelerate ions from 2 to $178 \mathrm{keV} / \mathrm{u}$. Continuous ion beams from ECRIS are converted into bunched beams of $26 \mathrm{MHz}$; this frequency is at least 1 order of magnitude higher than the required frequency. The linear accelerators of the ISAC facility use a prebuncher at $11.8 \mathrm{MHz}$, the third subharmonic of the RFQ frequency [8]. A single-gap multiharmonic prebuncher of $11.8 \mathrm{MHz}$ has been installed there not only for the efficient acceleration by the $35.4 \mathrm{MHz}$ RFQ linac but also for the experimental requirements. RIBs having a bunch spacing of 85 or $170 \mathrm{~ns}$ can be delivered by coupling the beam chopper placed downstream of the RFQ linac to the prebuncher. 


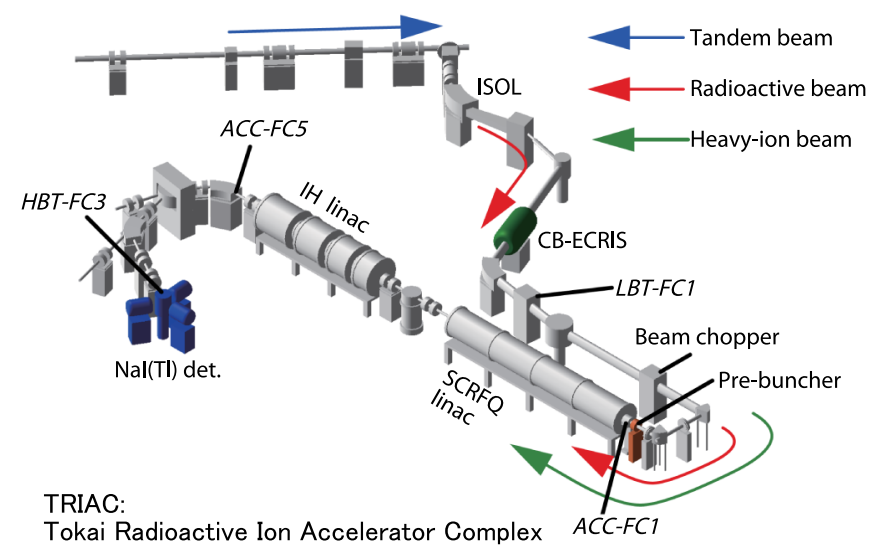

FIG. 1. Schematic layout of the TRIAC facility. Heavy-ion beams of stable isotopes are produced in an $18 \mathrm{GHz}$ ECR ion source (CB-ECRIS), which is also used as a charge state breeder for radioactive ion beams from the isotope separator on-line (ISOL). Then the ions are injected into the linac complex in the direction of the green arrow. The beam-bunching system, including the beam chopper and the prebuncher, is also indicated in this figure together with some of the Faraday cups for beam current measurement (location names in italics). The $\mathrm{NaI}(\mathrm{Tl})$ detectors for the ${ }^{12} \mathrm{C}(\alpha, \gamma)$ experiments are colored blue.

Therefore, we planned to construct a system consisting of a prebuncher and a beam chopper upstream of the linacs; however, the available area between ECRIS and SCRFQ is not sufficient to accommodate these elements. Hence, a novel technique has been developed to downsize the system without causing a significant beam particle loss. In this report, we describe the prebunching system. This includes the principle of the prebuncher, a beam simulation in both the prebuncher and the SCRFQ, the design of the beam chopper, and results of the system test. In addition, we present some experimental results related to the ${ }^{12} \mathrm{C}(\alpha, \gamma){ }^{16} \mathrm{O}$ reactions.

\section{PREBUNCHER}

\section{A. Principle of non- $\boldsymbol{\pi}$ mode beam bunching}

It is well known that a sawtooth voltage pattern is suitable for efficient beam bunching. A pseudo sawtooth pattern can be realized, for example, by an appropriate combination of the fundamental, second harmonic, and third harmonic sine-wave components with a single-gap buncher [8]; however, the effect of the leakage electric field is not negligible in the case of a large $\beta \lambda$, where $\beta$ is the particle velocity in units of light speed and $\lambda$ is the wavelength of the applied rf wave. In reality for the $2 \mathrm{MHz}$ bunching, the $\beta \lambda$ is about $30 \mathrm{~cm}$, where the attenuation of the leakage field becomes large and the fields after the gap are not averaged out for the moving particles. The resultant bunching is quite different compared to the original one. A multiple two-gap buncher system has a negligible leakage field, and it is ideal for sawtooth wave bunching; however, this system occupies a larger space and is more expensive than a single-gap buncher.

The sawtooth wave bunching is not possible using a single two-gap buncher, because some harmonic wave potentials cannot be applied in the simple $\pi$ mode operation. In this mode, we set the interval between two gaps as $\beta \lambda / 2$. Therefore, the second harmonic wave, for example, is not applied in pseudo sawtooth wave operation up to the third order. This practical difficulty can be overcome by applying modern digitized waveform techniques if we operate the buncher in a non- $\pi$ mode.

Let us express the gap interval as $\beta \lambda \theta / 2 \pi$, where $\theta$ is the phase difference between two gaps. The effective voltages applied to the first and the second gap up to the third order harmonics are given by

$$
\begin{aligned}
V_{1 \mathrm{st}} & =\sum_{n=1}^{3} V_{n} \cdot \sin \left(n \omega t+\varphi_{n}\right), \\
V_{2 \mathrm{nd}} & =\sum_{n=1}^{3}-V_{n} \cdot \sin \left(n \omega t+\varphi_{n}+n \theta\right),
\end{aligned}
$$

where $n, V_{n}$, and $\varphi_{n}$ denote the harmonic number of the $\mathrm{rf}$ waves, the effective potential seen by the particles, and the phase of each harmonic wave, respectively. The fundamental frequency of the rf wave is denoted by $\omega$. It is noted that the phase of each harmonic wave is treated independently in the non- $\pi$ mode operation, while all harmonic waves are usually in phase in the $\pi$ mode operation. At the second gap, the sign of the electric potential $V_{n}$ is reversed and the phase $\varphi_{n}$ advances by $n \theta$. The effective synthesized acceleration voltage ( $\left.V_{\text {total }}\right)$ based on $V_{1 \text { st }}$ and $V_{2 \text { nd }}$ is then expressed as

$V_{\text {total }}=\sum_{n=1}^{3} V_{n} \cdot 2 \sin (n \theta /-2) \cdot \sin \left[n \omega t+\varphi_{n}+(n \theta+\pi) / 2\right]$,

which becomes the pseudo sawtooth wave when $V_{n}$ and $\varphi_{n}$ are set as

$$
V_{n}=a_{n} \cdot \frac{V}{2 \sin (n \theta / 2)}, \quad \varphi_{n}=-\frac{n \theta+\pi}{2}, \quad(n=1,2,3),
$$

as shown in Fig. 2. In the present case, the coefficient $\left[a_{n}=(-1 / 3)^{n-1}\right]$ of each harmonic potential component [9] is different from that $\left[a_{n}=(-1)^{n-1} / n\right]$ in the Fourier expansion, because it yields a relatively smooth curve within this approximation range.

The actual gap voltage of each harmonics should be estimated by taking the corresponding transit time factor (TTF) into account. This factor for the $n$th harmonic wave can be estimated by the following approximation:

$$
\mathrm{TTF}=\frac{\beta \lambda}{g \pi} \sin (\pi g / \beta \lambda)
$$




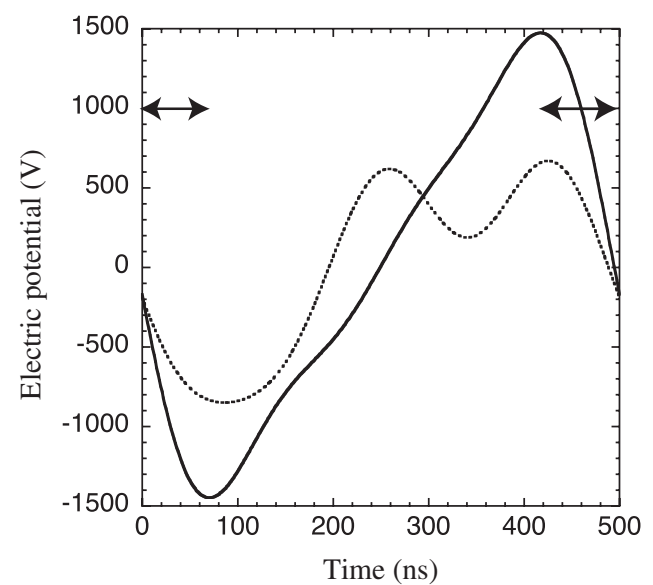

FIG. 2. The rf voltage waveform applied to the drift tube. The effective potentials seen by the particles passing through the first gap $\left(V_{1 \mathrm{st}}\right.$, dotted line $)$ and the second gap $\left(V_{\text {total }}=V_{1 \mathrm{st}}+V_{2 \text { nd }}\right.$, solid line) are indicated. The negative potentials indicate particle deceleration. The two areas from 0 to $70 \mathrm{~ns}$ and from 420 to $500 \mathrm{~ns}$, indicated by arrows, denote out-of-bunching phases.

where $g$ and $\lambda$ denote the gap spacing of the electrodes and the wavelength for the $n$th harmonic wave, respectively. When we assume the effective gap space to be $20 \mathrm{~mm}$, TTF's for the first, second, and third harmonics have almost the same values: $0.993,0.973$, and 0.940 , respectively. Actual values are expected to be a little bit larger than the approximated values due to the narrow gap spacing of around $10 \mathrm{~mm}$.

In this non- $\pi$ mode operation, the electric power $(P)$ can be expressed as

$$
P \propto \frac{1}{T} \int_{0}^{T} V_{1 \mathrm{st}}^{2} d t=\frac{1}{2} \sum_{n=1}^{3} V_{n}^{2},
$$

where $T$ is the period of the fundamental wave frequency. There are suitable phase differences giving minimum power at 140 and 220 degrees. For our case of $2 \mathrm{MHz}$ bunching at $\theta=140$ degrees, the gap interval becomes $12.05 \mathrm{~cm}$, which is sufficiently small for installation. Using a digitized waveform generator, we can easily generate an arbitrary wave, as expressed by Eq. (1).

In the above description, we restrict the harmonic components up to the third order. However, in principle, Eq. (2) can be expanded in the same manner for cases including higher order wave components.

By using a digitized waveform generator, it becomes possible to generate a pseudo sawtooth wave in a different way than the present one. This type of buncher has been reported in Refs. [10,11], where the sawtooth wave was generated by applying a parabolic wave on a drift tube of the two-gap buncher. When the gap interval is slightly different from the $\beta \lambda$, each beam particle experiences the difference between the voltages at a slightly shifted phase. The resultant wave pattern is nearly equal to the sawtooth. This buncher has a unique feature, in principle, that the effective bunch region becomes about $90 \%$ of the period [11], although in reality the ideal parabolic waveform might be distorted largely by the transfer functions of the cables and amplifier [12], since the higher harmonics to
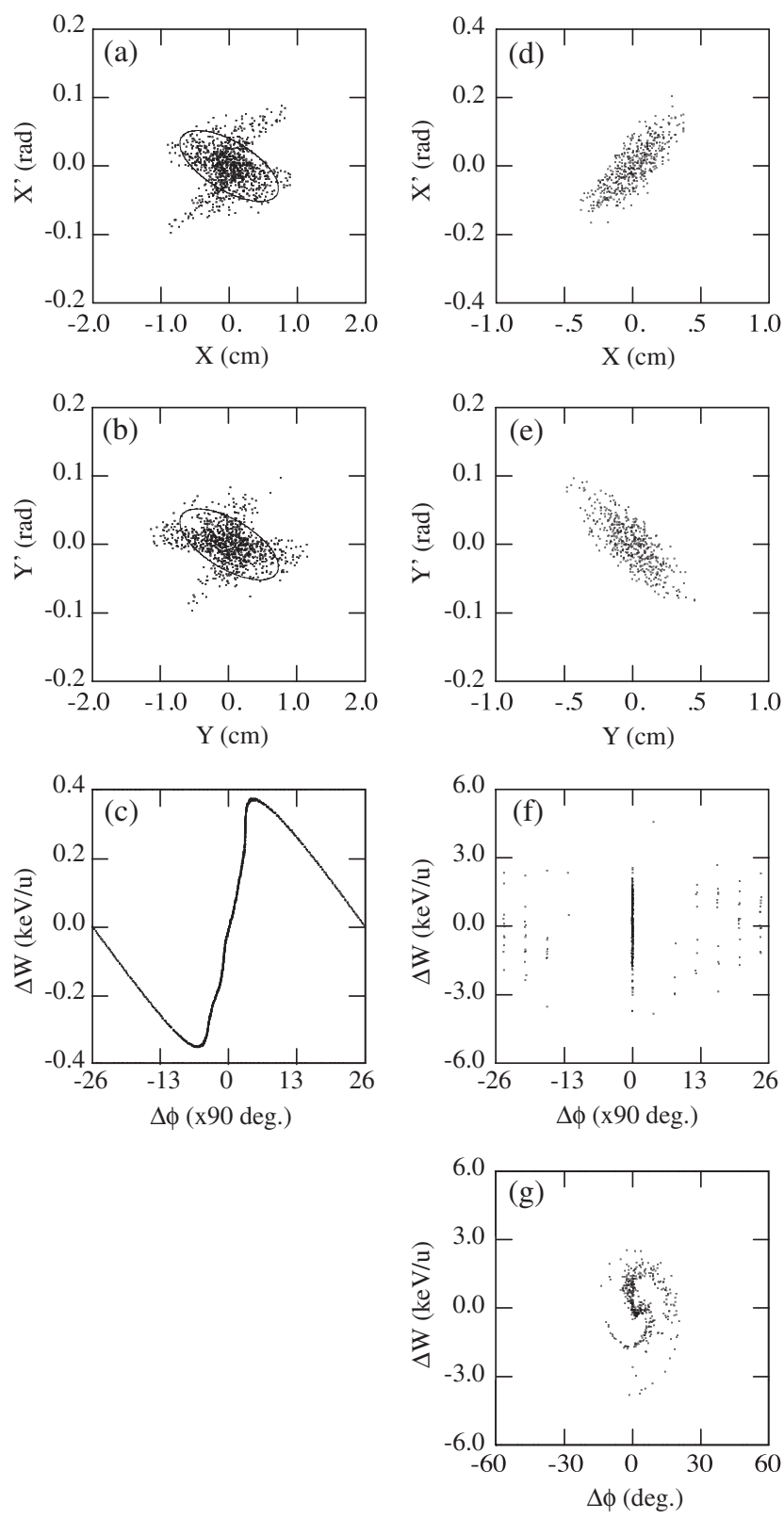

FIG. 3. Longitudinal and transverse emittances of a simulated beam at the entrance and exit of the SCRFQ after the prebuncher. Figures (a) and (b) show the transverse emittances at the entrance of the SCRFQ in terms of the $x-x^{\prime}$ and $y-y^{\prime}$ planes, respectively. Ellipses in these figures show design emittances ( $291 \pi \mathrm{mm} \mathrm{mrad)}$ ). Parts (d) and (e) show the same emittances as (a) and (b) at the exit of the SCRFQ. The vertical and horizontal axes of these figure are $x^{\prime}$ or $y^{\prime}$ in radians and $x$ or $y$ in $\mathrm{cm}$, respectively. Parts (c) and (f) show the longitudinal emittances $\Delta \phi-\Delta W$ at the entrance and exit of the SCRFQ in degrees and $\mathrm{keV} / \mathrm{u}$, respectively, which correspond to phase and particle energy. Part (g) is a magnified view of (f) around the central microbunch. 
the fundamental frequency are included in the parabolic wave. For generating efficient sawtooth waveforms, it is worthwhile to further study both approaches, e.g., the multisinusoids and the parabolic wave.

Before installing the prebuncher, we simulated the trace of ions from the entrance of the prebuncher to the exit of the SCRFQ. The particle motion in the prebuncher was calculated using the TRACEP code $[13,14]$ and the particle motion in the SCRFQ was calculated with the PARMTEQ code [15]. In the simulation by the TRACEP code, the TTF including the radial evolution of the longitudinal component of the electric field was taken into account to find out the realistic beam profile. Figure 3 shows the calculated beam profiles at the entrance and the exit of the SCRFQ. One thousand test particles were tracked in this simulation. The charge to mass ratio $(q / A)$ of the test particles is $q / A=1 / 4$ and the gap interval is $12.05 \mathrm{~cm}$ for $2 \mathrm{MHz}$ bunching.

The prebuncher is located $92.6 \mathrm{~cm}$ upstream of the SCRFQ. As shown in Fig. 3(c), the simulated longitudinal emittance at the entrance of the SCRFQ indicates successful sawtooth bunching in the central region in the range of \pm 2340 degrees, corresponding to $500 \mathrm{~ns}$. The energy distribution is slightly larger than the acceptable limit of the $\operatorname{SCRFQ}(\Delta E \sim \pm 0.2 \mathrm{keV} / \mathrm{u})$ for optimum bunching. This is attributed to the geometrical setup of the facility, as shown in Fig. 1. On the basis of the simulated emittance upstream of the SCRFQ [Fig. 3(a)-3(c)], we performed a particle simulation using PARMTEQ for the acceleration process inside the SCRFQ [Figs. 3(d)-3(g)].
A microstructure of the accelerated bunches is visible in Fig. 3(f) outside the central bunched region. Forty-one percent of the tested particles are gathered at the center, corresponding to a bunching gain of 5.4, which is defined as the ratio of beam particles of the center bunch with the prebuncher to those without the prebuncher. On the other hand, around $10 \%$ of the particles remain in the neighboring bunches. The longitudinal beam emittance in the center bunch can be seen in Fig. 3(g), a magnified view of Fig. 3(f). The relatively low bunching efficiency $(41 \%)$ is attributed to the large energy distribution of the bunched particles in front of the SCRFQ. A higher efficiency could be realized if the prebuncher would be installed at a distance that is sufficiently far from the SCRFQ; the necessary bunching voltage would also decrease.

\section{B. Setup}

The prebuncher, consisting of two gaps with a single drift tube, as shown in Fig. 4, is not a resonator; hence, the rf voltage is directly applied to the drift tube. The gap interval can be varied from 15 to $7 \mathrm{~cm}$ to match the bunching conditions in the frequency range of 2 to $4 \mathrm{MHz}$. The drift tube consists of a cylindrical electrode and two ring electrodes, each of which is electrically connected to the cylindrical electrode by eight skewer sticks, as shown in Fig. 4. The size of each gap is set as $10 \mathrm{~mm}$, which is sufficiently small for the expected $\beta \lambda$ of the beams we consider here.

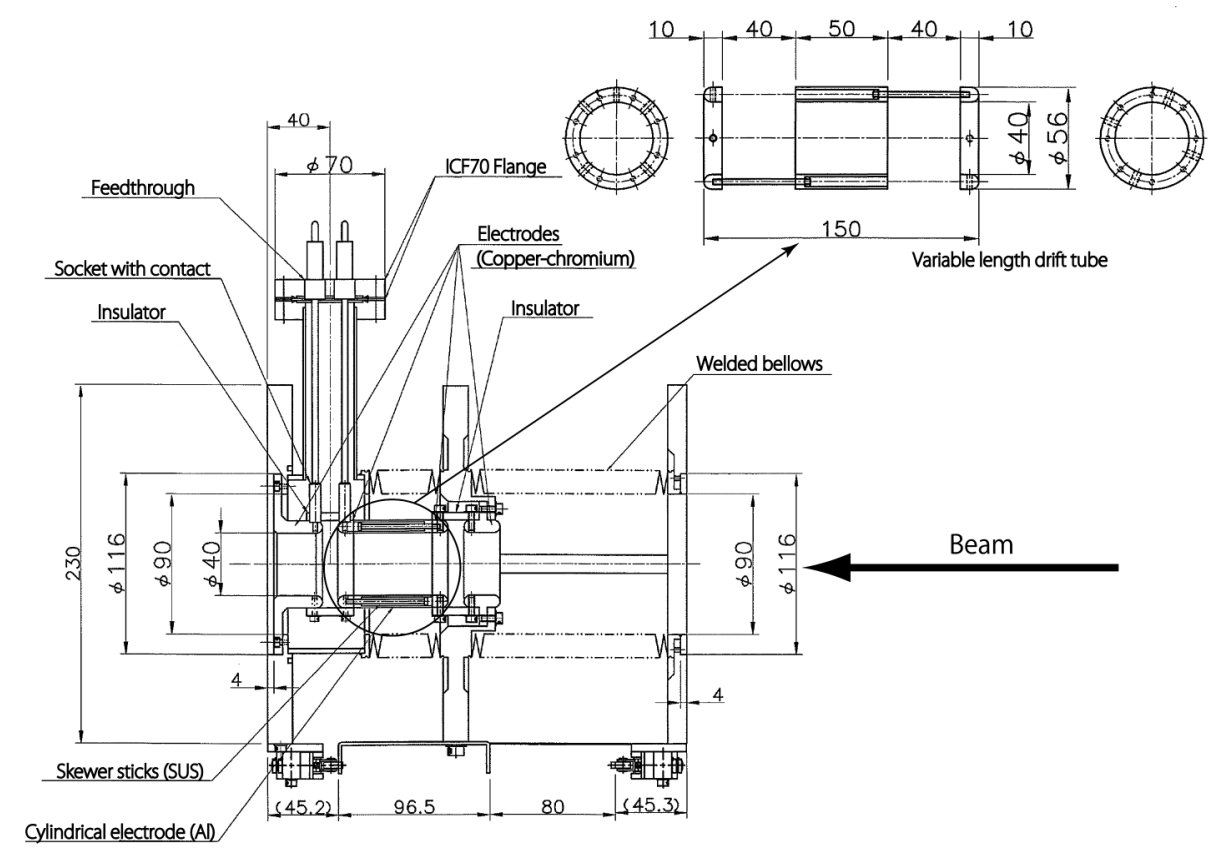

FIG. 4. Cross-section view of the prebuncher. The beam particles come from the right side. The details of the drift-tube structure are shown in the upper right corner of the figure. There are eight sticks of stainless steel placed cylindrically at each end of the aluminum electrode. They are connected to copper-chromium ring electrodes to form a variable length drift tube. The first and second electrodes, which are perpendicular to the beam direction, are isolated from each other in order to form the first electric potential gap. These electrodes are attached to the movable flange. The rf voltage is directly applied to the drift tube via the feedthrough. 
An impedance converter (T010-6046A [16]) is placed between an rf amplifier (50 $\Omega$, T145-6036AJA [17]) and the prebuncher $(450 \Omega)$, as shown in Fig. 9. The rf electric power up to $1.0 \mathrm{~kW}$ is acceptable for this converter. A single rf amplifier driven by an arbitrary function generator (AFG) [18] is sufficient for realizing a complicated waveform, as shown in Fig. 2. The maximum gain of this amplifier is $60 \pm 1.5 \mathrm{db}$ in the frequency range of 0.5 to $15 \mathrm{MHz}$. In our case, a single waveform to be generated by the AFG consists of 10000 sample points for one bunch cycle, for example spanning over $500 \mathrm{~ns}$ corresponding to $2 \mathrm{MHz}$ bunching frequency. The time step of the data points is 10 times shorter than the AFG sampling step $(\sim 0.5 \mathrm{~ns})$ to realize an accurate output, since the AFG sampling step cannot be controlled by the user and it does not always skip the same data point in every cycle. Iteratively the sample values are adjusted so that the measured rf output waveform approximates the ideal expected shape. These iterations are necessary, because the gain and phase of the rf amplifier gradually change as functions of frequency. The ideal waveform is obtained by taking the TTF values for each of the effective harmonic potentials $\left[V_{n}\right.$ in Eq. (3)] into account.

\section{BEAM CHOPPER}

\section{A. Principle of a multilayer beam chopper}

There are out-of-bunch phases in the pseudo sawtooth prebuncher as shown in Fig. 2. Beam particles in these phases will contribute to the unwanted background. A suitable chopping device for these particles is needed for the pulsed beam operation. An rf deflector after the first stage accelerator is usually referenced as a beam chopper [19].

The rf deflector reduces the number of background beam particles by applying an electric potential to the parallel plate electrodes. The required voltage is usually determined based on the velocity of accelerated particles. A sine-wave potential is usually adopted for this type of deflector, since most accelerated particles are bunched by the acceleration frequency of the first stage accelerator. Hereafter this beam bunch is called a "microbunch." In this chopping scheme, it is important to suppress the neighboring microbunches with respect to the ones that are expected to pass the chopper unaffected. The required deflecting potential becomes high, as the chopping frequency becomes low compared to the acceleration frequency.

When we consider an rf deflector just after the SCRFQ linac, the effective electrode length will be $100 \mathrm{~mm}$ according to the time separation of $38 \mathrm{~ns}$ between the microbunches. If we assume the electrode gap and deflection angle are $30 \mathrm{~mm}$ and $10 \mathrm{mrad}$, respectively, the required deflection voltage becomes $4.1 \mathrm{kV}$ due to the particle kinetic energy of $178 \mathrm{keV} / \mathrm{u}$. For the present case of $2 \mathrm{MHz}$ beam bunching, 12 microbunches should be deflected clearly in every 13 microbunches. This requires an rf potential of $36 \mathrm{kV}$ at maximum and a $36 \mathrm{kV}$ DC bias to be consistent with the cosine-wave deflection reported in Ref. [20]. Such a high voltage deflector needs too much space, so it cannot be set up in the TRIAC facility.

Hereafter, let us consider a chopper before the accelerator. To achieve sharp chopping, the electrode length of the single parallel plate should be as small as $1 / 10$ of $\beta \lambda / 2$. For the case of $2 \mathrm{MHz}$ bunching, the required length is $10 \mathrm{~mm}$. When the gap is assumed to be $40 \mathrm{~mm}$ due to the actual beam diameter of approximately $30 \mathrm{~mm}$, the effective field length is estimated to be as long as $\sim 100 \mathrm{~mm}$ around the electrode, which is about $1 / 3$ of the $\beta \lambda$. Moreover, the required deflection voltage becomes as high as $2 \mathrm{kV}$. Therefore, such a simple structure is not acceptable.

In order to shorten the effective field length and to suppress the deflection voltage, we considered a small gap deflector. When the gap is $1.9 \mathrm{~mm}$, the required voltage to one side of the electrode pair becomes only $100 \mathrm{~V}$ with reference to the grounded other side. Then we can stack several of these deflector pairs vertically, e.g., perpendicular to the beam direction to cover the beam aperture. This way the effective electric field can be minimized to extend only around $1.5 \mathrm{~mm}$ outside the deflector structure due to the alternate ordering of the applied potentials between positive voltage and the ground. This is shown in Fig. 5, where we estimate the field distributions at the medium plane of the beam line for the single gap deflector and the multilayer beam chopper. In this estimation, the thickness of each electrode in the multilayer beam chopper is $0.1 \mathrm{~mm}$ and the electric field in each device is normalized so that the sum of the calculated fields is equal to one.

The multilayer beam chopper deflects the beam particles upward and downward depending on the alternate direction of the electric field. The relatively low deflection voltage makes it easy to realize an application of the rectangular shape potential as mentioned below, although a certain fraction of beam particles would be lost by hitting the electrodes.

\section{B. Setup}

The newly developed beam chopper was located $4 \mathrm{~m}$ upstream of the prebuncher, as shown in Fig. 1. It consists of 19 electrode plates stacked in parallel as shown in Fig. 6. The gap between the $0.1 \mathrm{~mm}$ thick plates is $1.9 \mathrm{~mm}$, supported by polyimide washers. The chopper can be remotely placed in or out of the beam position using a pneumatic linear actuator. Positive and ground potentials are alternately applied to the electrodes to achieve upward and downward vertical deflection.

Beam particles lost by the chopper electrodes were measured by observing the ${ }^{16} \mathrm{O}^{4+}$ beam current just upstream of the SCRFQ. The observed fraction of beam loss was around $14 \%$, which was 3 times larger than the geometrical value (5\%), which is the ratio of the sum 


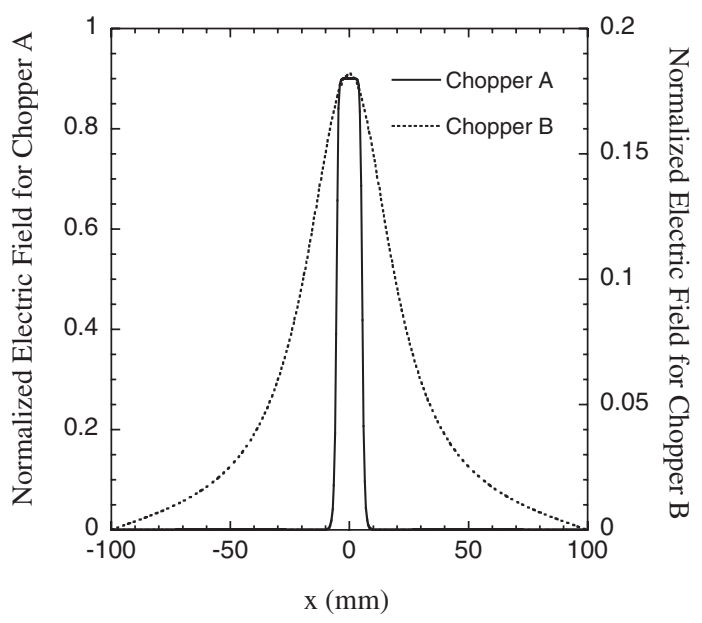

FIG. 5. Distribution of the normalized deflection electric field around the chopper. Solid and dashed lines indicate the field distributions for the multilayer beam chopper (Chopper A) and the single gap deflector (Chopper B), respectively. Each field strength is normalized so that the sum of the calculated fields is equal to one. The deflection angles for choppers A and B are the same for charged particles in the medium plane of the beam line. The length of the chopper electrodes is $10 \mathrm{~mm}$ for both chopper structures. The gaps for choppers A and B are 1.9 and $30 \mathrm{~mm}$, respectively.

of the plate thickness and the total height of the stack. This discrepancy can be explained by the finite angular distribution of the actual beam emittance in the vertical plane. Using a Faraday cup, the beam suppression was measured as a function of the chopping potential from zero to $+160 \mathrm{~V}$. The beam currents were normalized for the case of $0 \mathrm{~V}$ potential. In this measurement, a DC potential was applied. Figure 7 shows a suppression as good as $10^{-5}$ when the chopping potential is higher than $130 \mathrm{~V}$.

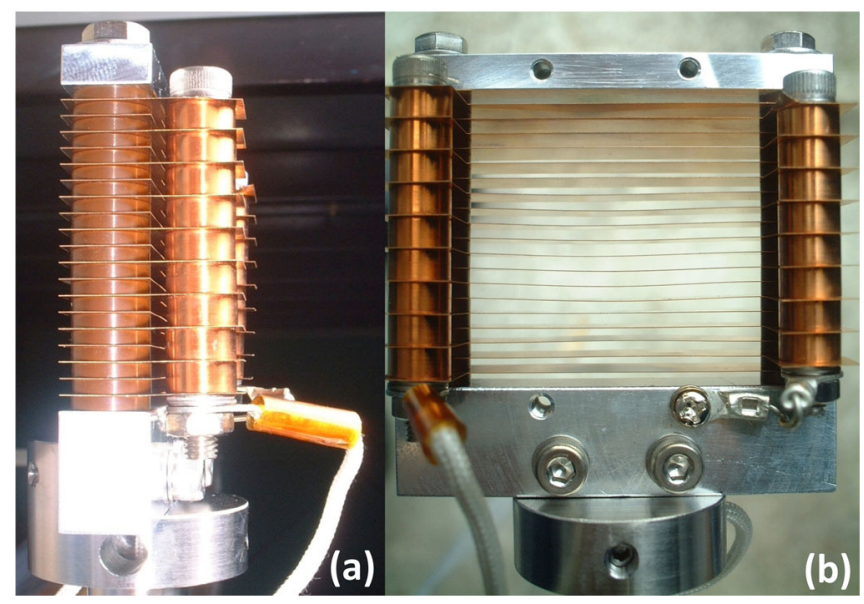

FIG. 6. Photographs of the multilayer beam chopper. (a) Side view of the chopper parallel to the beam; the beam goes from right to left. (b) Front view as seen by the beam particles entering the structure. The chopping voltage is applied on the left side pillar electrode through a shielded cable, while the right side pillar is grounded.

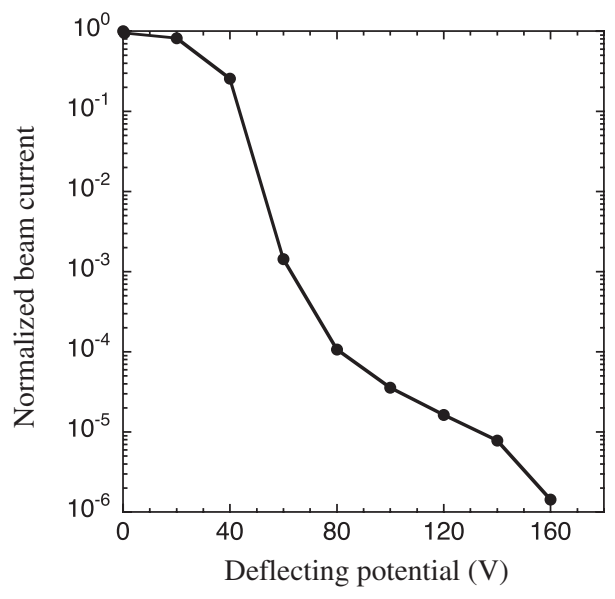

FIG. 7. Normalized beam current plotted as a function of the deflecting potential. The vertical axis indicates the normalized beam current compared to the reference situation at $0 \mathrm{~V}$ potential.

In the prebunching system, the chopper is operated under the rectangular shape potential with 2 to $4 \mathrm{MHz}$ frequency. This waveform is also generated by the AFG and amplified by the rf amplifier [21], as shown in Fig. 9. In this amplifier, the bias- $T$ circuit has been set externally to bias the bipolar rectangular wave $\left( \pm V_{p}\right.$ in pulse top) by the $\mathrm{DC}$ bias $\left(+V_{p}\right)$ for setting the $0 \mathrm{~V}$ potential. The circuit of this module is customized for accepting the DC bias of $100 \mathrm{~V}$ at maximum and the input rf power of $100 \mathrm{~W}$ at maximum. Moreover, a shaping circuit is installed between the rf amplifier and the chopper electrode in order to adjust the potential shape by reducing the electric ringing. The typical rise/fall time is $50 \mathrm{~ns}$, as shown in Fig. 8.

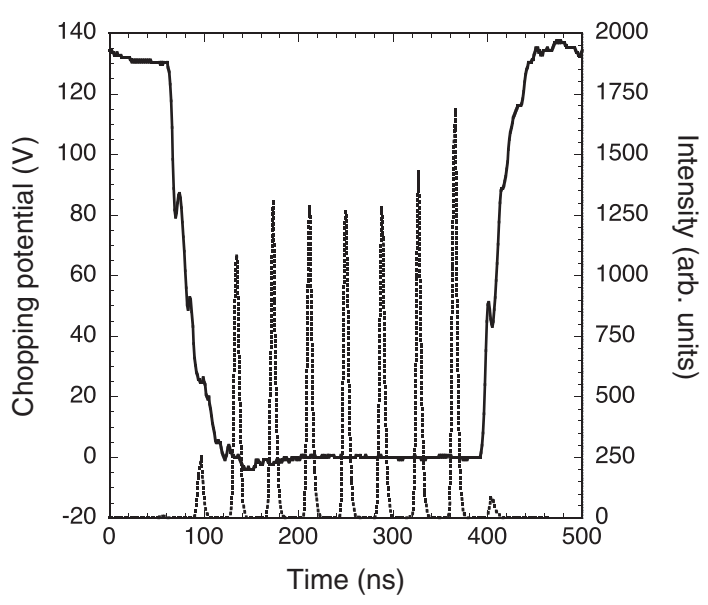

FIG. 8. The rectangular shaped deflecting potential applied to the chopper electrode is indicated by the solid line. This signal is monitored directly on the electrode. The applied potential is indicated by the vertical axis on the left-hand side. The time structure of chopped $\alpha$ beams after acceleration is shown by the dotted line. The observed beam intensity is indicated by the vertical axis on the right-hand side. 


\section{CONTROL OF PREBUNCHING SYSTEM}

Figure 9 shows the control circuit of the prebunching system connected to the accelerator rf control. An rf signal for the SCRFQ linac is generated by a signal generator with fundamental frequency, $f_{\mathrm{SG}}=25.9564 \mathrm{MHz}$. A logic signal $\left(f_{\text {trig }}\right)$ from a function generator is used as a trigger for all rf amplifiers of acceleration devices, such as the SCRFQ linac and the IH linac consisting of four independent tanks, under operation with various duty factors. The duration $\left(t_{d}\right)$ of the rf signal is variable in each controller of the acceleration device within the range of $t_{d}=$ 0.05-3.0 ms. Then, the duty factor of beam acceleration can be expressed as $t_{d} \cdot f_{\text {trig. }}$.

A coincident signal with $f_{\mathrm{SG}}$ and $f_{\text {trig }}$ is used as a trigger for the AFG module, with the timing synchronized to $f_{\mathrm{SG}}$. The AFG module generates waveforms, as expressed by Eq. (1), for the prebuncher at $\mathrm{CH} 1$ in Fig. 9 and rectangular

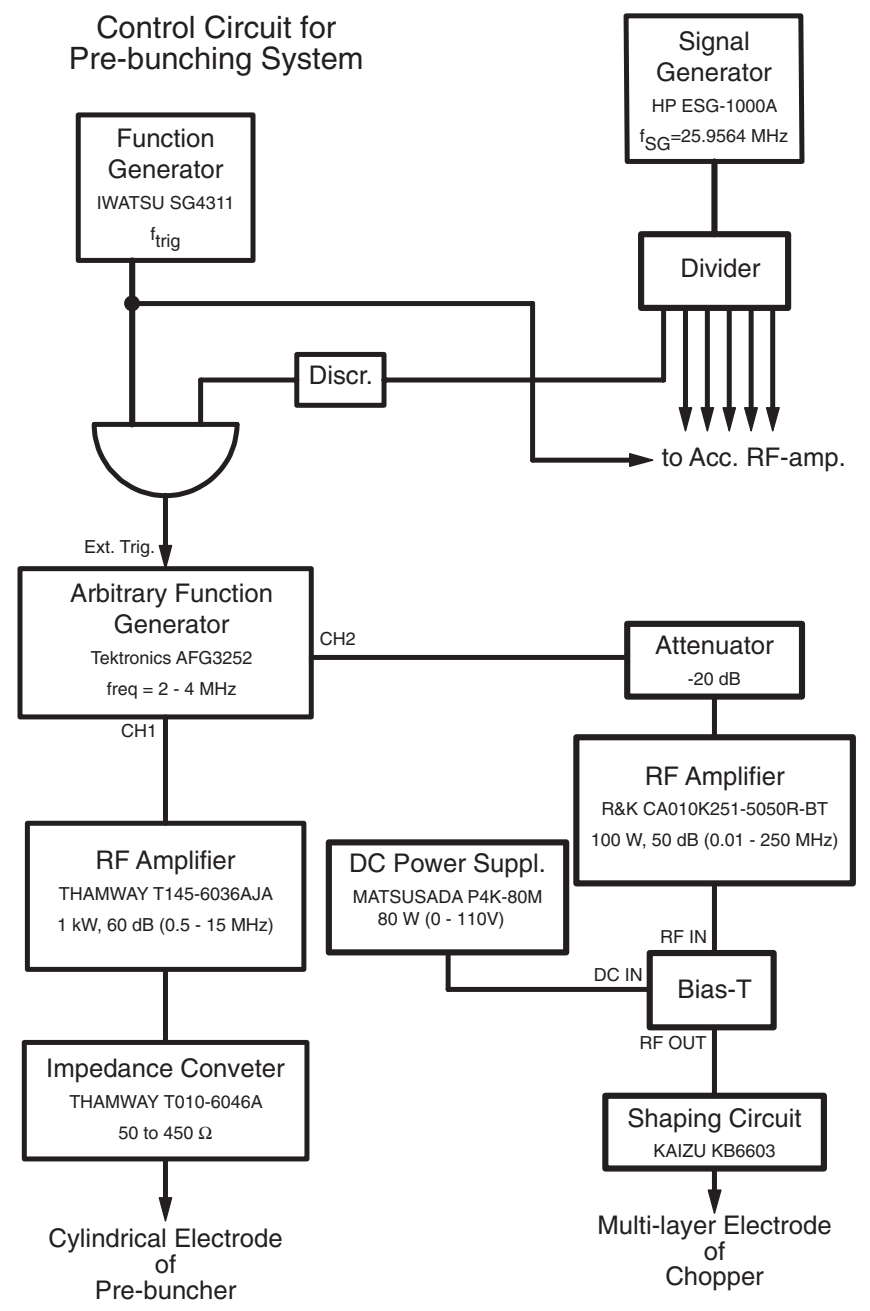

FIG. 9. Schematic diagram of the control circuit for the prebunching system. The parameters of the AFG generated waveform, such as the amplitude, delay timing to the external trigger, and repetition number for the unit wave in one cycle, can be changed remotely using the local network. waveforms for the chopper at $\mathrm{CH} 2$. The duration of each generated wave is adjusted to cover the beam acceleration period $\left(t_{d}\right)$ by changing the number of repetitions of the unit waveform from 2 to $4 \mathrm{MHz}$ in the AFG module. The start time of these waves can be adjusted to the timing of the actual beam microbunch by changing the timing delays independently.

The AFG is remotely controlled by a connection via a local area network so that iterative tuning can be performed by monitoring the beam profile. In a typical operation, the frequency of the trigger signal and the duration of the accelerator $\mathrm{rf}$ signal were $f_{\text {trig }}=350 \mathrm{~Hz}$ and $t_{d}=$ $1.7 \mathrm{~ms}$, respectively, indicating a duty factor $59.5 \%$. The repetition numbers (corresponding duration times) of the AFG waves for the prebuncher and chopper were 3600 $(1.8 \mathrm{~ms})$ and $4000(2 \mathrm{~ms})$, respectively, under the condition of $2 \mathrm{MHz}$ bunching.

\section{PERFORMANCE OF PREBUNCHING SYSTEM}

\section{A. Bunched $\alpha$ beams}

The performance was investigated using $\alpha$ beams of $1.1 \mathrm{MeV} / \mathrm{u}$. The beam currents were measured using Faraday cups in front of the chopper (LBT-FC1), just upstream and downstream of the linac complex (ACC-FC1 and ACC-FC5), and at the secondary target position (HBT$\mathrm{FC} 3$ ), around $8 \mathrm{~m}$ away from the ACC-FC5, as shown in Fig. 1. At the target position, the time structure was measured using a silicon detector, by detecting elastically scattered beam particles from a thin aluminum foil placed on the beam line.

The measured beam currents for different bunching modes are summarized in Table I: (A) acceleration without chopper and prebuncher, (B) acceleration with only prebuncher, and (C) acceleration with chopper and prebuncher. In these measurements, the duty factor of the accelerator is kept constant (33\%). The bunching frequency was set as $2 \mathrm{MHz}$, where 13 microbunches are included in one bunching cycle, as shown in Fig. 10(a). The number of beam particles in each microbunch would be the same. Hence, the beam current per microbunch without the chopper and prebuncher can be considered as $0.32 / 13 \mu \mathrm{A}=0.0246 \mu \mathrm{A}$.

In the first step, the time structure of the $\alpha$ beams was changed under the acceleration mode (B), as shown in Fig. 10(b), where beam particles in one bunching cycle were gathered in the central microbunch using the prebuncher. Its fraction was obtained as the ratio of the area of the central bunch to that of all the microbunches in one bunching cycle (79\% from this figure). Since the measured beam current in one bunching cycle can be considered as $0.17 \mu \mathrm{A}$ indicated in Table I, the normalized current of the central microbunch is estimated to be $0.17 \times 0.79=$ $0.134 \mu \mathrm{A}$. The bunching gain, defined as the normalized current ratio at the central microbunch for condition (B) to that for condition (A), becomes $0.134 / 0.0246=5.46$. 
TABLE I. Measured beam current in different acceleration modes.

\begin{tabular}{lcccc}
\hline \hline & \multicolumn{3}{c}{ Beam current $(\mu \mathrm{A})$} \\
Acceleration mode & LBT-FC1 & ACC-FC1 & ACC-FC5 & HBT-FC3 \\
\hline A: $w / o$ chopper and prebuncher & 2.0 & 1.58 & 0.38 & 0.32 \\
B: with prebuncher & 1.9 & 1.6 & 0.25 & 0.17 \\
C: with chopper and prebuncher & 2.0 & 0.6 & 0.18 & 0.12 \\
\hline \hline
\end{tabular}

The time width of the observed central microbunch was 12 ns in FWTM, which is sufficiently small, as compared to the required value of $20 \mathrm{~ns}$. However, there exists a considerable number of background beam particles in the region around the central microbunch. The fraction is larger than $20 \%$, which does not satisfy the experimental condition.
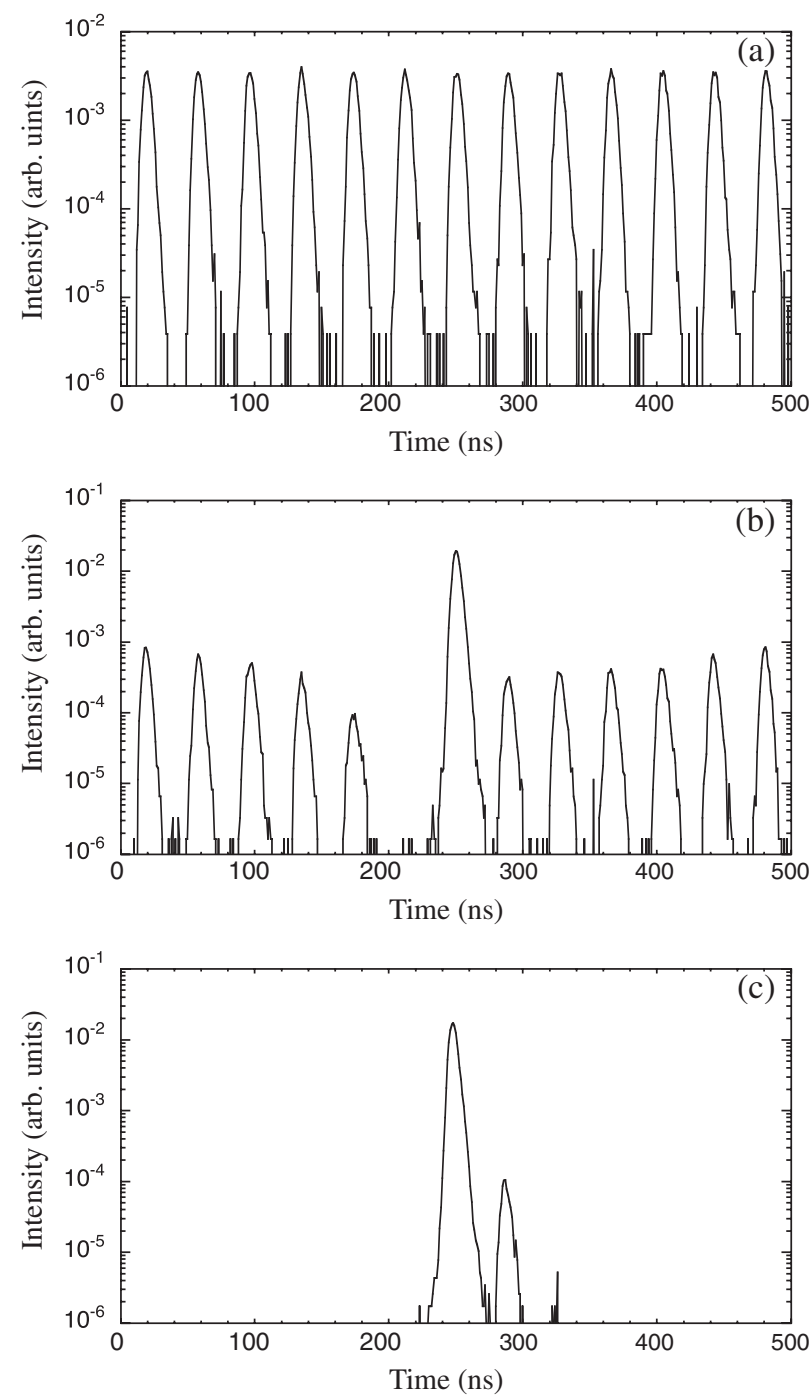

FIG. 10. Time structures of $\alpha$ beams under different acceleration modes. Parts (a), (b), and (c) correspond, respectively, to the conditions A (without chopper and prebuncher), B (with prebuncher), and $\mathrm{C}$ (with chopper and prebuncher). These conditions are described in the text.
In the second step, the chopper was operated together with the prebuncher. The chopping fraction in one bunching cycle was $33 \%$ in units of the full width at half maximum (FWHM), as shown in Fig. 8, and the start timing of the deflection potential wave was adjusted not only to optimize the bunching gain but also to minimize the number of background particles. The obtained time structure of the chopped $\alpha$ beams is shown in Fig. 8. Along with the prebuncher, the time spectrum was significantly improved, as shown in Fig. 10(c), where 99.4\% of the total beam particles in one bunching cycle are gathered in the central microbunch. Taking the measured beam current at HBT-FC3 into account, the normalized beam current at the central bunch is estimated as $0.119 \mu \mathrm{A}$. The resultant bunching gain becomes 4.85 in the same manner as for the first step. The slightly smaller value, as compared to the simulation (5.4), can be explained by the beam loss (14\%) due to the insertion of the beam chopper. The time width of the central bunch was 13 ns in FWTM, similar to condition (B). The number of background beam particles, especially over 250 ns just before the central microbunch, was well suppressed to $5 \times 10^{-5}$ of the number of bunched beam particles. This result fulfills the experimental condition.

\section{B. $\gamma$ ray background in ${ }^{12} \mathrm{C}(\alpha, \gamma){ }^{16} \mathrm{O}$ reactions}

The $\gamma$ decay from an $\alpha$-capture state to the ground state in ${ }^{16} \mathrm{O}$ has been measured using the anti-Compton $\mathrm{NaI}(\mathrm{Tl})$ spectrometer [7] with bunched $\alpha$ beams from TRIAC. The typical beam intensity on the target was $6 \mathrm{p} \mu \mathrm{A}$. The acceleration energy of the $\alpha$ particles was set as $701 \mathrm{keV} / \mathrm{u}$ and the characteristic energy of the $\gamma$ rays became $9.26 \mathrm{MeV}$. The operation parameters of the prebunching system are the same as mentioned in Sec. IV.

The target is enriched ${ }^{12} \mathrm{C}$ of $99.99 \%$ purity, with some ${ }^{13} \mathrm{C}$ contaminant. This isotope becomes a dominant neutron source through ${ }^{13} \mathrm{C}(\alpha, n)$ reactions. Neutrons with kinetic energy of less than $4.9 \mathrm{MeV}$ are emitted from the target. Gamma rays from ${ }^{127} \mathrm{I}(n, \gamma){ }^{128} \mathrm{I}$ and ${ }^{127} \mathrm{I}\left(n, n^{\prime} \gamma\right){ }^{127} \mathrm{I}$ with fast and thermal neutrons are induced in the $\mathrm{NaI}(\mathrm{Tl})$ detector located $0.3 \mathrm{~m}$ from the ${ }^{12} \mathrm{C}$ target. Gamma rays induced by prompt neutrons can be detected when the flight time of the neutrons exceeds $10 \mathrm{~ns}$, as continuous $\gamma$ rays ranging from 0 to $4.9 \mathrm{MeV}$ through ${ }^{127} \mathrm{I}\left(n, n^{\prime} \gamma\right){ }^{127} \mathrm{I}$ reactions and also as those ranging from 0 to $11.7 \mathrm{MeV}$ through ${ }^{127} \mathrm{I}(n, \gamma)^{128} \mathrm{I}$ reactions. On the other hand, discrete $\gamma$ rays around $6.83 \mathrm{MeV}$ (the neutron separation energy 


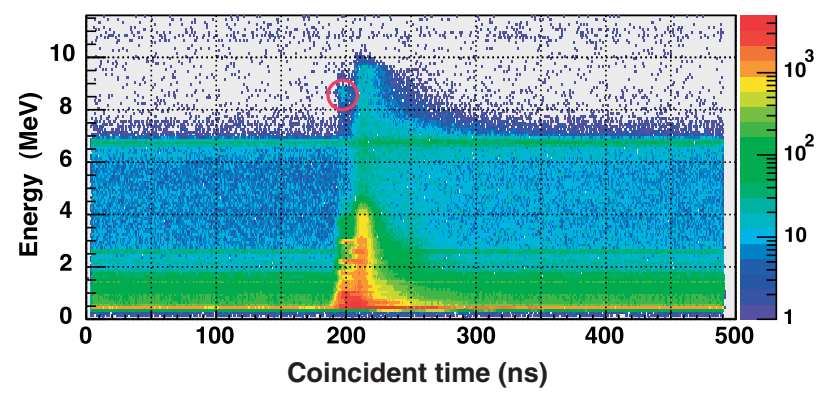

FIG. 11. Observed $\gamma$ ray correlations in energy and time for the ${ }^{12} \mathrm{C}(\alpha, \gamma){ }^{16} \mathrm{O}$ reactions using bunched $\alpha$ beams. The vertical and horizontal axes correspond to the observed $\gamma$ ray energy in $\mathrm{MeV}$ and coincident time between the $\gamma$ ray detection and the $\mathrm{rf}$ signal in nanoseconds, respectively. The intensities of the correlated events are denoted by different colors, as indicated by the scale on the right-hand side of the figure. The $\gamma$ ray events from the $\alpha$-capture state to the ground state in ${ }^{16} \mathrm{O}$ can be found inside the red circle.

of $\left.{ }^{128} \mathrm{I}\right)$ induced by ${ }^{127} \mathrm{I}(n, \gamma){ }^{128} \mathrm{I}$ with thermal neutrons can be observed anytime.

Figure 11 shows a measured correlation spectrum of the energies and the coincident times of the events detected by $\mathrm{NaI}(\mathrm{Tl})$ detectors. The trigger of the coincident time is the linac rf signal $(26 \mathrm{MHz})$. Characteristic $9 \mathrm{MeV} \gamma$ rays decaying from the $\alpha$-capture state in ${ }^{16} \mathrm{O}$ can be found at $200 \mathrm{~ns}$ as a part of prompt events, indicated in a red circle in the figure, whereas the intense $\gamma$ rays at $6.8 \mathrm{MeV}$ throughout the time region indicates $\gamma$ rays from ${ }^{127} \operatorname{In}(n, \gamma)$ reactions of thermal neutrons. Hence, the beam particles before prompt events are well smeared out by the prebunching system, and the prompt events are clearly separated from the induced $\gamma$ ray events at $210 \mathrm{~ns}$ and beyond, in spite of a considerable energy over $9 \mathrm{MeV}$ being deposited. The reaction cross section can be deduced accurately for the area of the $9 \mathrm{MeV} \gamma$ ray photopeak.

\section{SUMMARY}

A compact prebunching system was developed at the TRIAC facility for direct measurements of ${ }^{12} \mathrm{C}(\alpha, \gamma){ }^{16} \mathrm{O}$ reactions. This system consists of a two-gap prebuncher and a multilayer electrode beam chopper. It is confirmed that the system fulfills the performance requirements for the experiments. The bunching gain is 4.85 and the suppression factor of the background particles over $250 \mathrm{~ns}$ just before the bunched beam is better than $5 \times 10^{-5}$.

In the experiments for ${ }^{12} \mathrm{C}(\alpha, \gamma){ }^{16} \mathrm{O}$ reactions, decaying $\gamma$ rays from the $\alpha$-capture state can be easily assigned by suppressing a large amount of background $\gamma$ rays from neutron-induced reactions of ${ }^{127} \mathrm{I}$. The prebunching system has been successfully implemented to realize such a lowbackground environment.

Finally, using the non- $\pi$ mode beam-bunching principle based on the AFG and the broadband rf amplifier, we could realize a suitable beam bunching within a relatively small installation space. This type of beam buncher can be potentially employed in various beam handling techniques.

\section{ACKNOWLEDGMENTS}

The authors would like to thank Dr. A. Schnase at Japan Atomic Energy Agency for fruitful discussions on the nonsinusoidal wave buncher. This work was supported by a Grant-in-Aid for Scientific Research (A) (Grant No. 20244036) from the Japan Society for the Promotion of Science (JSPS).

[1] H. Miyatake, S. Arai, Y. Arakaki, Y. Fuchi, H. Ishiyama, S. C. Jeong, I. Katayama, K. Niki, T. Nomura, M. Okada, M. Oyaizu, Y. Takeda, M. H. Tanaka, E. Tojyo, M. Tomizawa, N. Yoshikawa, S. Abe, S. Hanashima, K. Horie, S. Ichikawa, H. Iimura, H. Ikezoe, T. Ishii, N. Ishizaki, A. Iwamoto, S. Kanda, M. Koizumi, M. Matsuda, S. Mitsuoka, Y. Nagame, T. Nakanoya, K. Nishio, I. Ohuchi, A. Osa, M. Oshima, M. Sataka, S. Takeuchi, H. Tayama, K. Tsukada, Y. Tsukihashi, and T. Yoshida, Nucl. Instrum. Methods Phys. Res., Sect. B 204, 746 (2003).

[2] S. Arai, Y. Arakaki, A. Imanishi, T. Katayama, H. Matsuda, K. Niki, M. Okada, Y. Takeda, E. Tojyo, N. Tokuda, M. Tomizawa, K. Yoshida, and M. Yoshizawa, CERN Report No. 96-07, 575, 1997.

[3] H. Ishiyama, T. Hashimoto, T. Ishikawa, Y.X. Watanabe, S. K. Das, H. Miyatake, Y. Mizoi, T. Fukuda, M. H. Tanaka, Y. Fuchi, N. Yoshikawa, Y. Hirayama, S.C. Jeong, T. Nomura, I. Katayama, S. Mitsuoka, K. Nishio, M. Matsuda, P. K. Saha, S. Ichikawa, H. Ikezoe, T. Furukawa, H. Izumi, T. Shimoda, and K. Nakai, Phys. Lett. B 640, 82 (2006).

[4] T. Hashimoto, H. Ishiyama, Y. X. Watanabe, Y. Hirayama, N. Imai, H. Miyatake, S.C. Jeong, M.-H. Tanaka, N. Yoshikawa, T. Nomura, S. Mitsuoka, K. Nishio, T. K. Sato, A. Osa, S. Ichikawa, M. Matsuda, H. Ikezoe, S. K. Das, Y. Mizoi, T. Fukuda, A. Sato, T. Shimoda, K. Otsuki, and T. Kajino, Phys. Lett. B 674, 276 (2009).

[5] R. Geller, Annu. Rev. Nucl. Part. Sci. 40, 15 (1990).

[6] S.C. Jeong, M. Oyaizu, E. Tojyo, H. Kawakami, H. Ishiyama, H. Miyatake, K. Enomoto, Y. Watanabe, I. Katayama, T. Nomura, M. Matsuda, A. Osa, and S. Ichikawa, Nucl. Instrum. Methods Phys. Res., Sect. B 204, 420 (2003).

[7] H. Makii, K. Mishima, M. Segawa, E. Sano, H. Ueda, T. Shima, Y. Nagai, and M. Igashira, Nucl. Instrum. Methods Phys. Res., Sect. A 547, 411 (2005).

[8] R.E. Laxdal, in Proceedings of the Particle Accelerator Conference, Chicago, IL, 2001 (IEEE, New York, 2001), p. 556.

[9] K. Ikegami and A. Goto, RIKEN Accel. Prog. Rep. 22, 213 (1988).

[10] K. Abrahamsson, G. Andler, L. Bagge, E. Beebe, P. Carlé, H. Danared, M. Engström, C. J. Herrlander, J. Hilke, J. Jeansson, A. Källberg, S. Leontein, L. Liljeby, A. Nilsson, A. Paal, A. Pikin, K.-G. Rensfelt, U. Rosengård, A. 
Simonsson, J. Starker, M. af Ugglas, H. Meuth, and A. Schnase, in Proceedings of the 4th European Particle Accelerator Conference, London, UK, 1994 (EPS-AG, London, UK, 1994), p. 380.

[11] W. Bräutigam, F.-J. Etzkorn, H. Jungwirth, and A. Schnase, IKP Annual Report No. 210 (1997).

[12] W. Bräutigam, R. Brings, R. Gebel, H. Jungwirth, A. Müller, and A. Schnase, IKP Annual Report No. 162 (1998).

[13] TRACEP is a useful simulation code for particle motion in the beam transport line connecting to the SCRFQ, since it has a similar FORTRAN common block data as the PARMTEQ code.

[14] S. Yamada, NIRS HIMAC Program manual No. 1 (1990).

[15] K. R. Crandall and T. P. Wangler, AIP Conf. Proc. 177, 22 (1988).
[16] Impedance converter, Report No. T010-6046A, THAMWAY Co. Ltd., Japan.

[17] RF Amplifier, Report No. T145-6036AJA, THAMWAY Co. Ltd., Japan.

[18] Arbitrary/Function Generator, Report No. AFG3252, TEKTRONIX, Inc.

[19] A. K. Mitra, Z.T. Ang, R. Hohbach, R. E. Laxdal, J. Lu, and R.L. Poirier, in Proceedings of the 21st International Linac Conference, Gyeongju, Korea, 2002 (Pohang Accelerator Laboratory, Pohang, Korea, 2002), p. 487.

[20] R. E. Laxdal, M. Pasini, and L. Root, in Proceedings of the 21st International Linac Conference, Gyeongju, Korea, 2002 (Ref. [19]), p. 407.

[21] RF Amplifier, Report No. CA010K251-5050R-BT, R\&K Co. Ltd., Japan. 